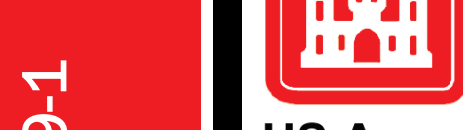

US Army Corps

of Engineers ${ }_{\circledast}$

Engineer Research and

Development Center

Environmental Consequences of Nanotechnologies

\title{
Determination of Fluorescence Emission and UV-Vis-NIR Absorbance for Nanomaterials Solution Using a HORIBA Scientific NanoLog ${ }^{\circledR}$ Spectrofluorometer
}

Qihua Wu, Kathryn Kremer, Stephen Gibbons, May 2019 and Alan J. Kennedy

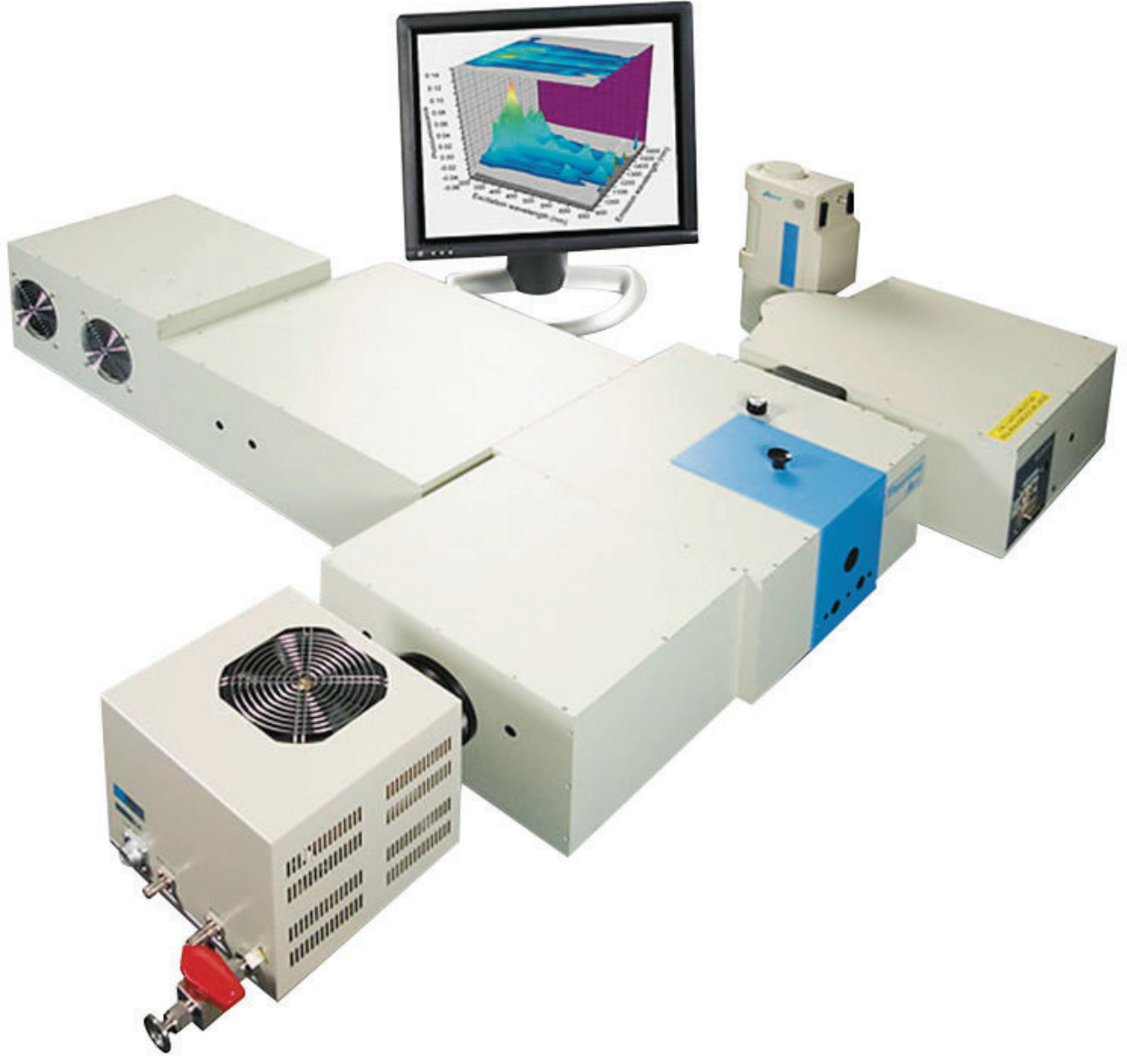


The U.S. Army Engineer Research and Development Center (ERDC) solves the nation's toughest engineering and environmental challenges. ERDC develops innovative solutions in civil and military engineering, geospatial sciences, water resources, and environmental sciences for the Army, the Department of Defense, civilian agencies, and our nation's public good. Find out more at www.erdc.usace.army.mil.

To search for other technical reports published by ERDC, visit the ERDC online library at http://acwc.sdp.sirsi.net/client/default. 


\title{
Determination of Fluorescence Emission and UV-Vis-NIR Absorbance for Nanomaterials Solution Using a HORIBA Scientific NanoLog ${ }^{\circledR}$ Spectrofluorometer
}

\author{
Alan J. Kennedy \\ Environmental Laboratory \\ U.S. Army Engineer Research and Development Center \\ 3909 Halls Ferry Road \\ Vicksburg, MS 39180-6199 \\ Qihua Wu, Kathryn Kremer, Stephen Gibbons \\ Brewer Science, Inc. \\ 2401 Brewer Drive \\ Rolla, MO 65401
}

Final report

Approved for public release; distribution is unlimited.

\author{
Prepared for U.S. Army Corps of Engineers \\ Washington, DC 20314-1000 \\ Under Work Unit 33143
}




\section{Abstract}

A fluorescence spectroscopy technique has been widely used for characterizing the fluorescence of nanomaterials. The technique provides fast and sensitive measurements that are applicable in many research and industrial areas. Obtained absorbance, excitation, and fluorescence emission spectra can be used to determine sample concentrations, binding kinetics of biomolecules, reaction mechanisms, and many more applications. This scientific operating procedure SOP provides general guidance for using fluorescence spectroscopy techniques with an instrument such as a HORIBA Scientific NanoLog ${ }^{\circledR}$ spectrofluorometer to determine fluorescence emission and UV-Vis-NIR absorbance of nanomaterials in solution. Procedures and recommendations of sample preparation, instrument preparation, sample analysis, and results analysis are briefly described. The procedure was tested on a variety of nanomaterial solutions.

DISCLAIMER: The contents of this report are not to be used for advertising, publication, or promotional purposes. Citation of trade names does not constitute an official endorsement or approval of the use of such commercial products. All product names and trademarks cited are the property of their respective owners. The findings of this report are not to be construed as an official Department of the Army position unless so designated by other authorized documents. 


\section{Contents}

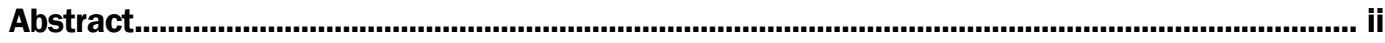

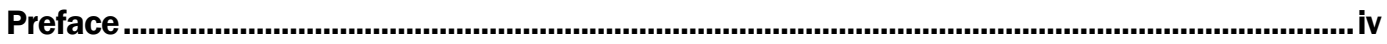

Unit Conversion Factors......................................................................................................................

Acronyms and Abbreviations .................................................................................................. vi

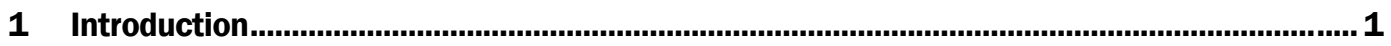

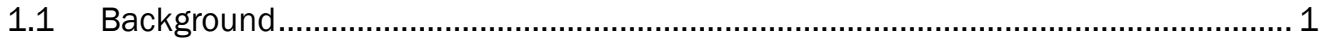

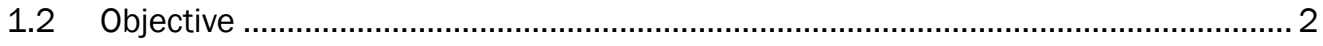

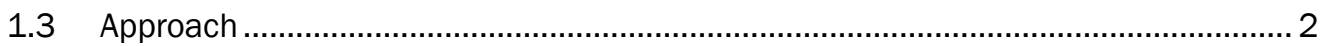

2 Terminology ....................................................................................................................... 4

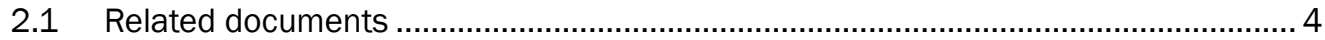

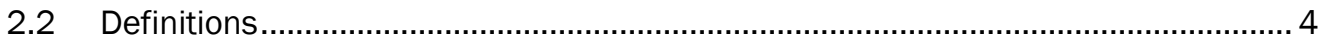

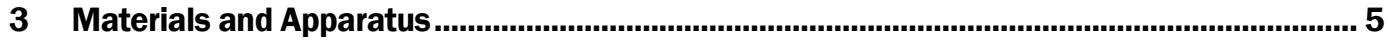

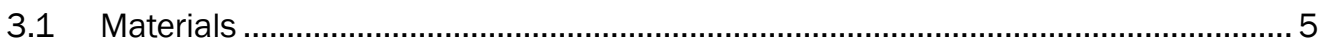

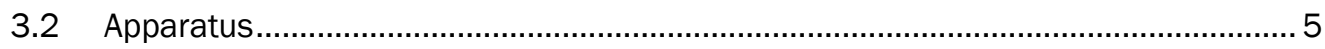

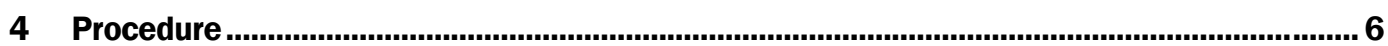

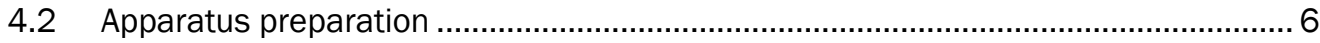

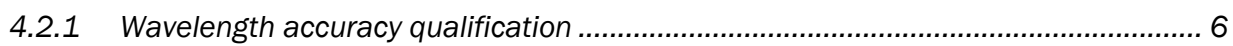

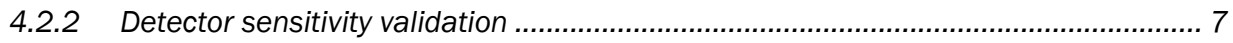

4.2.3 Other instrument qualification ........................................................................... 8

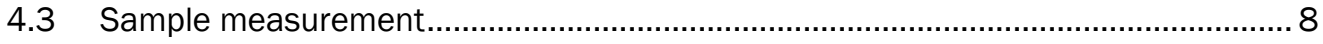

4.3.1 Fluorescence emission measurement................................................................ 8

4.3.2 UV-Vis-NIR absorbance measurement............................................................... 9

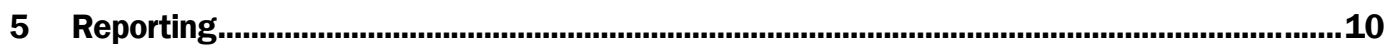

5.1 Analysis of fluorescence emission results ....................................................... 10

5.2 Analysis of absorbance results ............................................................................. 10

5.3 Quality Assurance (QA)/Quality Control (QC) considerations .................................. 10

References.

\section{Report Documentation Page}




\section{Preface}

This scientific operating procedure (SOP) is part of the "Advancing Carbon Nanomaterials-Based Device Manufacturing through Life Cycle Analysis, Risk Assessment and Mitigation" project, Work Unit 33143, directed by Dr. Rishi J. Patel, Senior Research Scientist at Missouri State University's Jordan Valley Innovation Center. The technical monitor was Mr. Jerry Miller (CEERD-EZT).

This work was coordinated by the Environmental Risk Branch (CEERDEPR) of the Environmental Processes Division (CEERD-EP), ERDC Environmental Laboratory (ERDC-EL). At the time of publication, Dr. Michael A. Rowland was the Acting Chief, CEERD-EPR, Mr. Warren P. Lorentz was Chief, CEERD-EP, and Dr. Elizabeth A. Ferguson, (CEERDEZT) was the Technical Director for Military Materials in the Environment. The Deputy Director of ERDC-EL was Dr. Jack E. Davis and the Director was Dr. Ilker R. Adiguzel.

COL Ivan P. Beckman was Commander of ERDC, and Dr. David W. Pittman was the Director. 


\section{Unit Conversion Factors}

\begin{tabular}{|l|l|l|}
\hline Multiply & By & To Obtain \\
\hline angstroms & 0.1 & nanometers \\
\hline degrees (angle) & 0.01745329 & radians \\
\hline feet & 0.3048 & meters \\
\hline gallons (U.S. liquid) & $3.785412 \mathrm{E}-03$ & cubic meters \\
\hline inches & 0.0254 & meters \\
\hline microinches & 0.0254 & micrometers \\
\hline microns & $1.0 \mathrm{E}-06$ & meters \\
\hline mils & 0.0254 & millimeters \\
\hline ounces (mass) & 0.02834952 & kilograms \\
\hline ounces (U.S. fluid) & $2.957353 \mathrm{E}-05$ & cubic meters \\
\hline pounds (mass) & 0.45359237 & kilograms \\
\hline quarts (U.S. liquid) & $9.463529 \mathrm{E}-04$ & cubic meters \\
\hline
\end{tabular}




\section{Acronyms and Abbreviations}

$\begin{array}{ll}\text { ASTM } & \text { American Society for Testing and Materials } \\ \Delta E & \text { energy difference } \\ \text { DI water } & \text { deionized water } \\ \text { DoD } & \text { Department of Defense } \\ \text { FCS } & \text { fluorescence correlation spectroscopy } \\ \text { FRET } & \text { fluorescence resonance energy transfer } \\ \text { IPA } & \text { isopropyl alcohol } \\ \text { NIR } & \text { near-infrared } \\ \text { NIST } & \text { National Institute of Standards and Technology } \\ \text { NISTIR } & \text { NIST Interagency/Internal Report } \\ \text { nm } & \text { nanometer } \\ \text { NPs } & \text { nanoparticles } \\ \text { QA } & \text { quality assurance } \\ \text { QC } & \text { quality control } \\ \text { QDs } & \text { quantum dots } \\ \text { SOP } & \text { scientific operating procedure } \\ \text { UV } & \text { ultraviolet } \\ \text { UV-Vis } & \text { ultraviolet-visible } \\ \text { UV-Vis-NIR } & \text { ultraviolet-visible-near-infrared } \\ & \text { in }\end{array}$




\section{Introduction}

\subsection{Background}

Fluorescence is the phenomenon that results when certain molecules that have absorbed light or electromagnetic radiation can re-emit light at another wavelength. All molecules have various energy states that refer to energy levels. For some compounds, when incident light hits the molecule in its ground state, the photon may be absorbed and the molecule is brought to its excited state by the absorbed energy. This process is called absorption and will only happen if the photon energy matches the energy gap between the ground and excited states of the molecule. When a molecule in its excited state returns to its ground state, the energy difference between the two states $(\Delta \mathrm{E})$ will be released from the molecule. One common process is that fluorescence light (with energy from the $\Delta \mathrm{E}$ ) will be emitted from the molecule. Since the molecule may return to different vibrational levels in the ground state, the emitted light will have various energies and frequencies/wavelengths. Fluorescence spectroscopy analyzes the frequency and intensity of light emitted from samples to determine the vibrational levels and identify the chemical structure.

A fluorescence spectrometer generally consists of an excitation light source, excitation/emission monochromator, and a detector. The detector is normally arranged perpendicularly to the incident light to suppress the interference from the excitation. Most fluorescence spectrometers have two basic measurement modes: the first mode is to detect emission spectra, during which the excitation wavelength is fixed and emission wavelength is scanned over a designed range; the second mode is to detect excitation spectra, during which the detected emission wavelength is fixed at maximum emission and the excitation wavelength is scanned. Many developments in fluorescence spectroscopy have been made, including fluorescence correlation spectroscopy (FCS), near-infrared (NIR) fluorescence, time-resolved fluorescence spectroscopy, and fluorescence microscopy (Yao et al. 2014).

With the emerging development of nanotechnologies, the combination of fluorescence and nanomaterials have been widely employed in various areas of chemistry, biology, and medicine-including drug delivery, disease diagnosis, pharmaceutical screening, medical imaging, and chemical 
sensing (Chinen et al. 2015; Howes et al. 2014; Lane et al. 2015; Iverson et al. 2013). Fluorescence nanoparticles (NPs) possess many special properties, including good biocompatibility, high quantum yield, great photostability, and narrower emission ranges (Clemens et al. 2005; Alivisatos et al. 1996; Canham 2007; Fan and Chu 2010). The use of fluorescence functional nanoparticles has enabled fast, sensitive, and reliable detection of biomolecular interaction at the micron- or even nanometer-size scale. For example, some semiconductor nanocrystals called quantum dots (QDs) (Smith and Nie 2004) have become an important part of approaches for bioanalysis such as fluorescence resonance energy transfer (FRET) detection. The conjugation of QDs and antibodies in FRETbased bioassays and biosensors have been extensively reported (Kagan et al. 1996; Wang et al. 2002; Willard et al. 2001). The use of fluorescence NPs has also enabled real-time imaging at the molecular level for many research and application areas (Weissleder et al. 2001).

\subsection{Objective}

This scientific operating procedure (SOP) is for determining fluorescence emission and ultraviolet-visible-near-infrared (UV-Vis-NIR) absorbance of nanomaterials in solution by using fluorescence spectroscopy.

This SOP does not provide a detailed method for a specific kind of nanomaterials; it provides a general guidance for most nanomaterial suspension solutions. The operation procedure for a HORIBA Scientific NanoLog ${ }^{\circledR}$ spectrofluorometer is demonstrated as an example.

Fluorescence correlation spectroscopy (FCS) analyzes hydrodynamic dimension and binding kinetics between donor and receptor, which can yield hydrodynamic radii, molecular diffusion coefficient, and chemical reaction kinetics (Nienhaus et al. 2013). Many other applications of and techniques for fluorescence spectroscopy combined with nanotechnologies exist but are beyond the scope of this SOP.

\subsection{Approach}

This document provides a SOP for determining the fluorescence emission and absorbance of nanomaterials in solution using fluorescence spectroscopy. This SOP was developed using a HORIBA Scientific NanoLog spectrofluorometer. This SOP is part of the scope of Task 2 "Materials process development and characterization," of the project 
"Advancing Carbon Nanomaterials-Based Device Manufacturing through Life Cycle Analysis, Risk Assessment and Mitigation.”

Advances of nanotechnologies have attracted tremendous interest in many fields such as next-generation electronics, medical diagnosis, disease treatment, high-sensitivity sensors, and many others (Webster 2006; Duncan and Gasper 2011; Lim et al. 2015; Yao et al. 2014). Many techniques have been utilized for characterizing physical and/or chemical properties of manufactured nanomaterials. Among these techniques, fluorescence spectroscopy has been extensively used to study absorbance and fluorescence emission of nanomaterials. It is an effective, rapid, informative, and sensitive technique for detecting certain chemical/ biological molecules, and it can also be used for studying diffusion and binding of biomolecules (Boukari and Sackett 2008; Sapsford et al. 2011). UV-Vis-NIR absorbance spectra obtained from fluorescence spectroscopy could provide characteristics of nanomaterials, including chemical structures, concentrations, and even binding kinetics with biomolecules (Jiang et al. 2005). Other useful information, including excitation wavelength, photo-stability, and quantum yield of nanomaterials, can also be measured. This SOP briefly describes the basic principle and operation of fluorescence spectroscopy to investigate UV-Vis-NIR absorbance and fluorescence emission of nanomaterials in solution. 


\section{Terminology}

\subsection{Related documents}

- ASTM D4763-06 Standard Practice for Identification of Chemicals in Water by Fluorescence Spectroscopy

- ASTM E2719-09 Standard Guide for Fluorescence - Instrument Calibration and Validation

- ASTM E388-04 Standard Test Method for Spectral Bandwidth and Wavelength Accuracy of Fluorescence Spectrometers

- NISTIR 7458 Standard Guide to Fluorescence - Instrument Calibration and Validation

- Instrument Operation Manual provided by instrument manufacturers (for example, HORIBA Scientific NanoLog spectrofluorometer)

\subsection{Definitions}

- Excitation: When a photon of an incident light is absorbed by a molecule, the absorbed energy pushes an electron from a ground state to an excited state. This only happens if the photon energy matches the energy gap between the ground and excited states of the molecule.

- Emission: A molecule in the excited state loses energy by means of emitting photons and returns to its ground state. In most cases, due to energy dissipation during the excited-state lifetime, the energy of the emitted photons is lower, and therefore of longer wavelength than the excitation photons. For some materials with high enough quantum efficiency (e.g., nanomaterials containing d-block or f-block elements), anti-stoke fluorescence can be generated at shorter wavelength than the incident light.

- Quantum yield: In fluorescence spectroscopy, the ratio of numbers of photons emitted to numbers of photons absorbed. Molecules with higher fluorescence quantum yield normally have stronger fluorescence emission.

- Photostability: The ability of molecules to remain stable when exposed to electromagnetic radiation or resist photochemical changes. 


\section{Materials and Apparatus}

\subsection{Materials}

- Nanomaterial sample solution

- Nanomaterial dispersion medium

- Deionized water (DI water)

- Isopropyl alcohol (IPA)

- Sample cuvette (quartz or ultraviolet (UV) glass, various suppliers and materials available)

- Reference standard (type and availability may vary, depending on instrument manufacturer)

- Band-pass filter (availability varies, depending on instrument manufacturer)

- Neutral density filter (availability varies, depending on instrument manufacturer)

- Pipettes

\subsection{Apparatus}

- A fluorescence spectrometer or spectrofluorometer (should contain a light source), a sample cell holder, excitation/emission monochromators, and an adequate detector.

- Various light sources can be used as excitation sources, including lasers, xenon lamps, or mercury vapor lamps. An excitation monochromator (or filter) is required for light sources that emit continuous spectrum (e.g., a xenon lamp).

- The detector used for the fluorescence spectrometer can be singlechanneled or multi-channeled in various types. The detector should be arranged perpendicularly to the excitation light source in most applications. A "front-face" measurement mode is available for some instruments when a sample has low emission; in this case, the detector is oriented at an angle of less than $90^{\circ}$ of incident light. 


\section{Procedure}

The procedure described below covers the generic practice for measuring fluorescence emission and absorbance of nanomaterials in solution. Some sections use the operation of a HORIBA Scientific NanoLog spectrofluorometer as the demonstration.

\subsection{Sample preparation}

The mass concentration of analytes can have a significant impact on test results. If the concentration is too low, sample absorbance and emission might be below the instrument detection limit. If the concentration is too high, it may cause several issues, including with the formation and interference of dimers/trimers, including the saturation of the detector. Depending on the quantum yield of the analyte, the appropriate sample concentration may be varied from $10^{-5} \mathrm{M}$ to $10^{-8} \mathrm{M}$. Follow the manufacturer's specification to dilute samples into suitable concentration. It is also recommended to prepare and test a series of sample solutions with different concentrations to find the appropriate concentration.

Small amounts of impurities or contaminants can dramatically affect test results. The sample cuvettes need to be cleaned and dried prior to conducting the measurements. The recommended method for cleaning the sample cuvettes is by rinsing them with a small amount of DI water and then alcohol (such as IPA), followed by DI water again. Some nanomaterials may have low photostability and are sensitive to light. To avoid photo-bleaching or photo-decomposition, it is recommended to store samples in the dark if they will not be tested immediately.

\subsection{Apparatus preparation}

This section describes the general procedure for apparatus preparation, qualification, and validation. Qualification and validation of instrument performance are required to ensure consistent performance and obtain reliable results. The operation of a HORIBA Scientific NanoLog spectrofluorometer is demonstrated as the example.

\subsubsection{Wavelength accuracy qualification}

Wavelength accuracy needs to be qualified on a daily basis prior to sample measurements. There are several methods for determining the accuracy of 
excitation and/or emission wavelength, including the use of atomic pen lamps (ASTM E388-04), rare earth element glass, holmium oxide standard, xenon source, or water Raman spectra. Different types of methods are applicable in various wavelength regions and have different resolutions. Follow the method that is recommended by the instrument manufacturer.

For a HORIBA Scientific NanoLog spectrofluorometer, water Raman signal is tested for wavelength accuracy qualification. Place a quartz cuvette containing 1.5 2 mL of DI water into the sample compartment and ensure that the instrument is in "Right angle" mode. Click on the "Experiment Menu" icon and choose "Spectra" when the dialog box appears. Select "Emission" mode from the "Experiment Type" dialog box. Then click on the "Load" button in the "Fluorescence Division Experiment Setup" dialog box. Select the default water Raman test method stored in the instrument method database to start. Once the test is completed, the "Peak Analyzer" dialog box will appear. Record the exact wavelength at maximum intensity (near $397 \mathrm{~nm}$ ), and calibrate the monochromator by clicking on the "Calibrate IHR 320" button.

\subsubsection{Detector sensitivity validation}

The response and sensitivity of the detector should also be checked daily before any sample is tested. The fluorescence signal of a reference standard is normally measured and compared to the background signal or a blank.

For a HORIBA Scientific NanoLog spectrofluorometer, an optical glass filter is used for detector signal verification in the UV-Vis-NIR region. Place an RG830 glass filter in the sample compartment near the signal exit portal at a $30^{\circ}$ angle. Click on the "Experiment Menu" icon and choose "Spectra" when the dialog box appears. Select "Emission" mode from the "Experiment Type" dialog box. Then click on the "Load" button in the "Fluorescence Division Experiment Setup" dialog box. Select the default laser glass test method stored in the instrument method database to start. The scanned wavelength ranges from $800 \mathrm{~nm}$ to $1700 \mathrm{~nm}$, with a slit width of $4 \mathrm{~nm}$ and an NIR ruled grating with groove density of 600 per millimeter and 1000-nm wavelength are used. Record the intensity value at $1053 \mathrm{~nm}$, and compare it with previous results. 


\subsubsection{Other instrument qualification}

Other qualifications or verifications are recommended based on the type and configuration of the fluorescence spectrometer. For example, lamp intensity verification can verify the energy level of the excitation light source. The spectral correction can reduce the deviation between the detected emission spectra and the true instrument-independent spectra (Gaigalas et al. 2009) and is recommended for nanomaterials with broad emission wavelength ranges. Refer to the manufacturer's instrument operation manual for a detailed procedure.

\subsection{Sample measurement}

The sample measurement procedure should follow the operation manual provided by instrument manufacturer. This section demonstrates a brief sample measurement procedure using a HORIBA Scientific NanoLog spectrofluorometer as an example.

\subsubsection{Fluorescence emission measurement}

Ensure all calibrations are completed and wait for at least 15 minutes after completion before sample measurement. Select the appropriate excitation wavelength, excitation slit width, emission slit width, and wavelength range of the emission scan. It is recommended to start the emission scan at a wavelength that is 10 to $15 \mathrm{~nm}$ higher than the excitation wavelength to avoid Rayleigh scatter. Check the fluorescence signal during sample measurement. Increase or decrease the emission slit width if the signal is too weak or is saturated. For samples with low photostability, enable the shutter function (if applicable) or reduce both excitation slit width and exposure time.

For a HORIBA Scientific NanoLog spectrofluorometer, open the "Main Experiment Menu" and select " $3 \mathrm{D}$ " from the options provided. Fill in the experimental conditions: excitation and emission wavelength, scan increments, slit width, and detector and gratings used. Ensure that the mirror setup is in "right-angle" mode. Add band-pass filter into the signal exit portal if needed. Click on "run" to collect the excitation-emission spectra. 


\subsubsection{UV-Vis-NIR absorbance measurement}

A HORIBA Scientific NanoLog spectrofluorometer is equipped with a second light source and InGaAs array detector for measuring UV-Vis-NIR absorbance. To make a ultraviolet visible (UV-Vis) (200 $800 \mathrm{~nm}$ ) absorbance measurement, select "tungsten lamp" as the light source and PMT as the detector. For absorbance in the NIR region (800 $1650 \mathrm{~nm}$ ), use xenon lamp as incident light and InGaAs detector. Select "axial" mode to enable absorbance measurement for both UV-Vis and NIR regions. A blank (solvent) sample needs to be tested before sample measurement. 


\section{Reporting}

\subsection{Analysis of fluorescence emission results}

Record the general profile of fluorescence emission spectra and wavelength at maximum intensity for each peak. Be sure to subtract the blank (solvent) spectrum from sample spectrum. For unknown samples, compare the emission spectrum with published data (for example, ASTM D4763.06). Be sure to compare spectra of samples that are tested in the same solvent and under similar conditions, such as excitation wavelength and emission scan range. Check peak positions, number of peaks, peak shapes, and the full width at half maximum intensity for identification of unknown components.

The obtained excitation spectrum can also be analyzed using same method as mentioned above. If the sample is sufficiently diluted, the excitation spectrum will fit closely with the absorbance spectrum. In this case, excitation spectra can also be used to help identify unknown components.

\subsection{Analysis of absorbance results}

Similar to analysis of fluorescence emission results, absorbance spectra and wavelength at maximum intensity for each peak should be recorded. Be sure to subtract the blank (solvent) spectrum from the sample spectra. For the concentration determination, use a pre-established calibration curve and the Beer-Lambert equation to calculate sample concentrations.

\subsection{Quality Assurance (QA)/Quality Control (QC) considerations}

For QA/QC purposes, a blank sample and a National Institute of Standards and Technology (NIST)-traceable standard should be measured before sample measurements and periodically checked during sample measurements. Refer to the manufacturer's specification for each standard. 


\section{References}

Alivisatos, A. P. 1996. Semiconductor clusters, nanocrystals, and quantum dots. Science 271(5251):933-937. DOI: 10.1126/science.271.5251.933.

Boukari, H., and D. L. Sackett. 2008. Fluorescence correlation spectroscopy and its application to the characterization of molecular properties and interactions. Methods in Cell Biology 84:659-678. https://doi.org/10.1016/S0091-679x(07)84021$\underline{0}$

Canham, L. T. 2007. Nanoscale semiconducting silicon as a nutritional food additive. Nanotechnology 18(18):185704.

Chinen, A. B., C. M. Guan, J. R. Ferrer, S. N. Barnaby, T. J. Merkel, and C. A. Mirkin. 2015. Nanoparticle probes for the detection of cancer biomarkers, cells, and tissues by fluorescence. Chemical Reviews 115(19):10530-10574. Doi: 10.1021/acs.chemrev.5boo321.

Clemens B., X. Chen, R. Narayanan, and M. A. El-sayed. 2005. Chemistry and properties of nanocrystals of different shapes. Chemical Reviews 105(4):1025-1102. Doi: 10.1021/cro30063a.

Duncan, R., and R. Gaspar. 2011. Nanomedicine(s) under the microscope. Molecular Pharmaceutics 8(6):2101-2141. Doi: 10.1021/mp200394t.

Fan, J., and P. K. Chu. 2010. Group IV nanoparticles: synthesis, properties, and biological applications. Small 6.19:2080.

Gaigalas, A. K., L. Wang, H. J. He, and P. DeRose. 2009. Procedures for wavelength calibration and spectral response correction of CCD array spectrometers. Journal of Research of the National Institute of Standards and Technology 114.4:215228. Doi: 10.6028/jres.114.015.

Howes, P. D., R. Chandrawati, and M. M. Stevens. 2014. Bionanotechnology. Colloidal nanoparticles as advanced biological sensors. Science 346(6205):1247390. DOI: 10.1126/science.1247390.

Iverson, N. M., P. W. Barone, M. Shandell, L. J. Trudel, S. Sen, F. Sen, V. Ivanov, E. Atolia, E. Farias, T. P. McNicholas, and N. Reuel. 2013. In vivo biosensing via tissue-localizable near-infrared-fluorescent single-walled carbon nanotubes. Nature Nanotechnology 8:873-880.

Jiang, X., J. Jiang, Y. Jin, E. Wang, and S. Dong. 2005. Effect of colloidal gold size on the conformational changes of adsorbed cytochrome c: probing by circular dichroism, UV-visible, and infrared spectroscopy. Biomacromolecules 6(1)1:4653. Doi: 10.1021/bmo49744l.

Kagan, C. R., C. B. Murray, M. Nirmal, and M. G. Bawendi. 1996. Electronic energy transfer in CdSe quantum dot solids. Physical Review Letters 76:1517. 
Lane, L. A., X. Qian, and S. Nie. 2015. SERS nanoparticles in medicine: From label-free detection to spectroscopic tagging. Chemical Reviews 115(19):10489-10529. Doi: 10.1021/acs.chemrev.5boo265.

Lim, E. K., T. Kim, S. Paik, S. Haam, Y. M. Huh, and K. Lee. 2015. Nanomaterials for theranostics: Recent advances and future challenges. Chemical Reviews 115(1):327-394. Doi: 10.1021/cr300213b.

Nienhaus, G. U., P. Maffre, and K. Nienhaus. 2013. Chapter Four - Studying the protein corona on nanoparticles by FCS. Methods in Enzymology 519:115-137. https://doi.org/10.1016/B978-0-12-405539-1.00004-X

Riley II, M. K. and W. Vermerris. 2017. Recent advances in nanomaterials for gene delivery-a review. Nanomaterials 7(5):94. https://doi.org/10.3390/nano7050094

Sapsford, K. E., K. M. Tyner, B. J. Dair, J. R. Deschamps, and I. L. Medintz. 2011. Analyzing nanomaterial bioconjugates: A review of current and emerging purification and characterization techniques. Analytical Chemistry 83(12):44531448. Doi: 10.1021/ac200853a.

Smith, A. M., and S. Nie. 2004. Chemical analysis and cellular imaging with quantum dots. Analyst 129(8):672-677.

Wang, S., N. Mamedova, N. A. Kotov, W. Chen, and J. Studer. 2002. Antigen/antibody immunocomplex from CdTe nanoparticle bioconjugates. Nano Letters 2(8):817822. Doi: 10.1021/nlo255193.

Webster, T. J. 2006. Nanomedicine: What's in a definition? International Journal of Nanomedicine 1(2):115-116.

Weissleder, R., and U. Mahmood. 2001. Molecular imaging. Radiology 219(2):316. https://doi.org/10.1148/radiology.219.2.r01ma19316

Willard, D. M., L. L. Carillo, J. Jung, and A. Van Orden. 2001. CdSe-ZnS quantum dots as resonance energy transfer donors in a model protein-protein binding assay. Nano Letters 1(9):469-474. Doi: 10.1021/nlo15565n.

Yao, J., M. Yang, and Y. Duan. 2014. Chemistry, biology, and medicine of fluorescent nanomaterials and related systems: New insights into biosensing, bioimaging, genomics, diagnostics, and therapy. Chemical Reviews 114(12):6130-6178. Doi: 10.1021/cr200359p. 
The public reporting burden for this collection of information is estimated to average 1 hour per response, including the time for reviewing instructions, searching existing data sources, gathering and maintaining the data needed, and completing and reviewing the collection of information. Send comments regarding this burden estimate or any other aspect of this collection of information, including suggestions for reducing the burden, to Department of Defense, Washington Headquarters Services, Directorate for Information Operations and Reports (0704-0188), 1215 Jefferson Davis Highway, Suite 1204, Arlington, VA 22202-4302. Respondents should be aware that notwithstanding any other provision of law, no person shall be subject to any penalty for failing to comply with a collection of information if it does not display a currently valid OMB control number. PLEASE DO NOT RETURN YOUR FORM TO THE ABOVE ADDRESS.

\begin{tabular}{l|l|l}
$\begin{array}{l}\text { 1. REPORT DATE } \\
\text { May } 2019\end{array}$ & $\begin{array}{l}\text { 2. REPORT TYPE } \\
\text { Final }\end{array}$ & 3. DATES COVERED (From - To) \\
\hline
\end{tabular}

\section{TITLE AND SUBTITLE}

Determination of Fluorescence Emission and UV-Vis-NIR Absorbance for

Nanomaterials Solution Using a HORIBA Scientific NanoLog ${ }^{\circledR}$

Spectrofluorometer

5a. CONTRACT NUMBER

\section{AUTHOR(S)}

Qihua Wu, Kathryn Kremer, Stephen Gibbons, and Alan J. Kennedy

\section{PERFORMING ORGANIZATION NAME(S) AND ADDRESS(ES)}

U.S Army Engineer Research and Development Center

Environmental Laboratory

3909 Halls Ferry Road, Vicksburg, MS 39180-6199

\section{SPONSORING/MONITORING AGENCY NAME(S) AND ADDRESS(ES)}

10. SPONSOR/MONITOR'S ACRONYM(S)

Headquarters, U.S. Army Corps of Engineers

Washington, DC 20314-1000

11. SPONSOR/MONITOR'S REPORT NUMBER(S)

\section{DISTRIBUTION/AVAILABILITY STATEMENT}

Approved for public release; distribution is unlimited.

\section{SUPPLEMENTARY NOTES}

\section{ABSTRACT}

A fluorescence spectroscopy technique has been widely used for characterizing the fluorescence of nanomaterials. The technique provides fast and sensitive measurements that are applicable in many research and industrial areas. Obtained absorbance, excitation, and fluorescence emission spectra can be used to determine sample concentrations, binding kinetics of biomolecules, reaction mechanisms, and many more applications. This SOP provides general guidance for using fluorescence spectroscopy techniques with an instrument such as a HORIBA Scientific NanoLog ${ }^{\circledR}$ spectrofluorometer to determine fluorescence emission and UV-Vis-NIR absorbance of nanomaterials in solution. Procedures and recommendations of sample preparation, instrument preparation, sample analysis, and results analysis are briefly described. The procedure was tested on a variety of nanomaterial solutions.

\section{SUBJECT TERMS}

Fluorescence emission, UV-Vis-NIR absorbance, nanomaterial, fluorescence spectroscopy

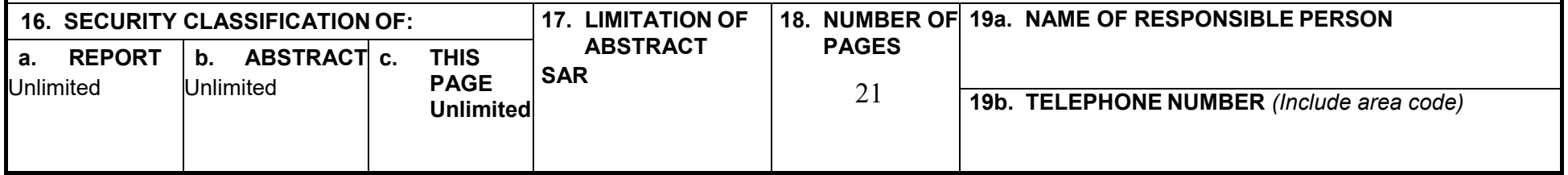

